157 SURVEY ON USE OF CAFFEINE IN APNOEA OF PREMATURITY IN NEONATAL UNITS ACROSS ENGLAND

doi:10.1136/archdischild-2012-302724.0157

D Abraham, P Rajagopal, E Curtis, A Manzoor, M Ahmed. Paediatrics, Queens Hospital, Burton upon Trent, UK

Background Apnoea of prematurity (AOP) is a significant clinical problem in premature infants and is almost universal in infants $<1000 \mathrm{~g}$ at birth. Caffeine has emerged as the methylxanthine of choice to treat AOP. Although it is commonly used, there is no unified consensus or guideline on its use in NNUs in England.

Aim To study the current practice of caffeine use in AOP at NNUs in England.

Methods A telephonic survey of level 3 and level 2 units in England was conducted, using a standardised questionnaire, over November and December, 2011.

Results Out of 52 units surveyed, 48\% were level 3 units. All units used caffeine for treatment of AOP (base $60 \%$ and citrate $40 \%$ of units). $92 \%$ of units have written guidelines on caffeine use. Caffeine was started by $47 \%$ of units based on gestational age, regardless of symptoms.[IS3] All units used a loading dose, which varied between 5 and $25 \mathrm{mg} / \mathrm{kg}$ (median of $10 \mathrm{mg} / \mathrm{kg}$ ) for caffeine base and 15 to $20 \mathrm{mg} / \mathrm{kg}$ (median of $20 \mathrm{mg} / \mathrm{kg}$ ) for citrate. The maintenance dose varied between $2.5-6 \mathrm{mg} / \mathrm{kg} /$ day (median of $5 \mathrm{mg} / \mathrm{kg} /$ day) for base and $5-12 \mathrm{mg} / \mathrm{kg} /$ day (median of $5 \mathrm{mg} / \mathrm{kg} /$ day) for citrate. Caffeine levels were routinely performed by $7 \%$ of units. Caffeine was discontinued between 30 to 36 weeks gestation.

Discussion Our survey depicts that practice of caffeine use varies significantly across NNUs in England. The results from this survey could be used as a footing for further data collection, for formulation of a uniform guideline maximising the utilisation of this extensively studied drug.

\section{A SYSTEMATIC REVIEW OF PHARNMACOKINETICS (PK) OF DOBUTAMINE FOR USE IN NEONATES AND CHILDREN}

doi:10.1136/archdischild-2012-302724.0158

${ }^{1}$ GS Shah, ${ }^{2} \mathrm{D}$ Crook, ${ }^{3} \mathrm{H}{ }^{4}$ Rojas-Anaya, H Rabe. 'Paediatric Academic Department, Brighton and Sussex Medical School; ${ }^{2}$ Clinical Investigations and Research Unit, ${ }^{3}$ Paediatric Academic Department, Brighton and Sussex University Hospitals; ${ }^{4}$ Paediatric Academic Department, Brighton and Sussex University Hospitals, Brighton and Sussex Medical School, Brighton, UK

Background Dobutamine has been used off-label in newborns and children for treating haemodynamic insufficiency for over 20 years. As preparation for a large randomised study to achieve Paediatric Use Marketing Authorisation for dobutamine in newborns we performed a structured literature review of PK data.

Methods Structured searches were conducted using the electronic databases Medline and Embase. Search terms included dobutamine, infant, newborn, paediatric/pediatric, prematurity, child, infant, low birth weight infant, preschool child, school child, adolescent, pharmacokinetics, clinical pharmacology. Data was extracted based on pre-defined criteria decided by the team.

Main results Six of eleven papers emerging from this search met our inclusion criteria. These reported dobutamine PK data in a combined total of 70 children (age range 0 days-22 years, 13 newborns, 27-42 weeks gestation), with infusion rates ranging from $0.5-20$ $\mu \mathrm{g} / \mathrm{kg} / \mathrm{min}$. Five papers found that the infusion rate was positively correlated to plasma dobutamine concentration. Dobutamine clearance rates showed great variability between individuals (range 35.1$482.2 \mathrm{~mL} / \mathrm{kg} / \mathrm{min}$ ). Four papers found that clearance did not vary with infusion rate, suggesting first order kinetics, although one paper $(n=12)$ showed a significantly negative relationship $(p<0.001)$ of dobutamine clearance to steady-state plasma concentration (dobutamine dosage $2-15 \mu \mathrm{g} / \mathrm{kg} / \mathrm{min}$ ).
Conclusions The current dobutamine PK data is difficult to interpret due to inhomogeneity and variability of patients' age and conditions, dobutamine dosages and study designs. High quality prospective PK data -especially in newborns- is urgently required prior to our large randomised study.

\section{PHARMACOKINETICS (PKS) OF LEVOSIMENDAN (LEVO) AND INTERMEDIATE METABOLITES (OR-1855 AND OR- 1896) IN NEWBORNS UNDERGOING CARDIOVASCULAR SURGERY WITH CARDIOPULMONARY BYPASS (CPB)}

doi:10.1136/archdischild-2012-302724.0159

${ }^{1} \mathrm{~A}$ Pellicer, ${ }^{2} \mathrm{R}$ Lubomirov, 'MC Bravo, 'P López-Ortego, ' $\mathrm{J}$ Pérez-Rodríguez, '1 Quero, ${ }^{1} \mathrm{~F}$ Cabañas. 'La Paz University Hospital, Department of Neonatology; ${ }^{2}$ Department of Clinical Pharmacology, La Paz University Hospital and Clinical Pharmacology Center, School of Medicine, Universidad Autónoma de Madrid. IdiPAZ., Madrid, Spain

Background and aims LEVO is a novel inodilator developed to treat heart failure. Biotransformation of LEVO in the intestinal tract gives rise to intermediate metabolites with prolonged beneficial haemodynamic effects. There are no data on LEVO PKs in neonates. We aim to investigate LEVO and intermediate metabolites PKs in newborns undergoing CPB.

Methods Eleven infants received step-wise dose increase of LEVO $(0.10,0.15,0.20 \mathrm{mg} / \mathrm{k} / \mathrm{min})$ delivered as i.v. continuous infusion, starting before CPB up to $48 \mathrm{~h}$ post-surgery. Eleven blood samples per subject were collected up to day 14 post-infusion started. Samples were quantified by HPLC-MS/MS. Non-compartmental methods were used for PK parameters. Median (IOR) values are reported. Results Area under the curve (AUC, ng*h/mL) OR-1855 plasma concentration [1717.10 (930.38-3756.41)] was 2.3- and 8.2-fold higher than LEVO [742.10 (527.23-1046)] and OR-1896 [209.78 (99.54-275.36)], respectively. LEVO clearance (CL, L/h/k) was 0.67 (0.44-1.0). OR-1855 maximum concentration (Cmax, $\mathrm{ng} / \mathrm{ml}$ ) was 5.2-fold higher than OR-1896 [18.5 (10.44-33.25) vs. 3.58 (2.94-4.38)]. OR-1896 and OR-1855 Cmax were respectively achieved $2 \mathrm{~h}$ before and $120 \mathrm{~h}$ after LEVO infusion stopped. LEVO CL increased and AUC decreased with postnatal age, explaining $66.23 \%$ $(p=0.023)$ and $34.51 \%(p=0.047)$ of their respective variance. LEVO AUC and pre-surgery antibiotics explained $38.89 \%(\mathrm{p}=0.016)$ and $26.68 \%(p=0.035)$ of OR-1855 AUC variance, respectively. Use of additional diuretics to furosemide explained $27.21 \%(\mathrm{p}=0.025)$ of OR-1896 AUC. No other covariates influenced LEVO or metabolites PKs.

Conclusions This study describes the pharmacokinetic profile of LEVO and intermediate metabolites in newborns as well as covariates explaining a significant part of their variance.

\section{LONGTERM OUTCOME AFTER LIVER TRANSPLANTATION: PSYCHOSOCIAL AND COGNITIVE ISSUES}

doi:10.1136/archdischild-2012-302724.0160

KH Schulz. University Medical Center Hamburg-Eppendorf, Hamburg, Germany

Psychosocial outcome in liver transplanted children was primarily investigated in terms of health related quality of life (HRQOL). In children this multidimensional construct additionally accentuates domains like school, family, and peers as well as physical and cognitive-emotional development.

Although organ transplantation is lifesaving, recipients trade a terminal illness for a chronic syndrome with good organ function in most cases, however. Nevertheless, restoration of organ function does not involve return to a normal life. It is characterized by fear of organ failure and complications, side effects of the medication, developmental deficits, and psychiatric comorbidities. Liver transplanted children show a poorer HRQOL compared with the healthy population, equal to or better than in children with other chronic diseases. Factors 
associated with decreased HROOL include: comorbidity, older age at transplantation, medication side effects, and parental conflict. Two specific problems in pediatric organ transplantation are adherence to medication and transition from pediatric to adult transplant care. Early disease onset, poor nutritional status, growth deficits, and longer duration of illness prior to transplant have been identified as factors contributing to an adverse cognitive development of these children.

Studies are heterogeneous regarding operationalization of HRQOL, study design, length of follow-up, and age of the children. There are only few prospective multi-center studies, which should be encouraged in future research including specific internationally accepted validated instruments.

Against the background of a new era of immunosuppressive therapy (steroid minimization, individualized therapy), a better long-term outcome in these children could be expected.

\section{CHILDREN AS DONORS: A NATIONAL PEDIATRIC INTENSIVE CARE STUDY TO ASSESS PROCUREMENT OF ORGANS AND TISSUES}

doi:10.1136/archdischild-2012-302724.0161

MJ Siebelink, MJIJ Albers, PF Roodbol, HBM van de Wiel. University of Groningen, University Medical Center Groningen, Groningen, The Netherlands

Objectives Shortage of size-matched organs and of tissues is the key factor limiting transplantation in children. Empirical data on the procurement process in children is sparse. This study aimed to gain insight into the recognition of potential pediatric donors in the Netherlands and the procurement process.

Methods A national retrospective cohort study in the Dutch pediatric intensive care units. The records of 683 deceased children were analyzed by two independent donation experts and procurement process data were compared with the national protocol.

Results From 2003 thru 2006, 74 (11\%) of the deceased children were found to have been suitable for organ donation and 132 (19\%) for tissue donation. Sixty-two (84\%) potential organ donors had been correctly identified; parental consent had been obtained and donation effectuated in 26/62 children (42\%). Sixty-three potential tissue donors (53\%) had been correctly identified; parental consent had been obtained and donation effectuated in $17 / 63$ children (27\%). Conclusion Recognition of pediatric organ donors by medical professionals is acceptable; recognition of tissue donors may be improved. Efforts to address the shortage of organs and tissues for transplantation in children should focus on the gap between recognition of donors and parental consent. We suggest such studies should not only assess the process itself, i.e. the competencies of the professional staff (micro-level) but also the influence of legislation, societal views on donation by children, and the potential relevance of children's views on donation (macro-level).

\section{CONGENITAL CHLORIDE DIARRHEA: A SINGLE CENTRE EXPERIENCE WITH 43 CHILDREN}

doi:10.1136/archdischild-2012-302724.0162

'SM Shabib, ${ }^{2}$ Al Al Mehaedib, ${ }^{1} \mathrm{HM}$ Nazer, ${ }^{1} \mathrm{AA}$ Abbad. ${ }^{1}$ Pediatrics; ${ }^{2}$ King Faisal Specialist Hospital \& Research Centre, Rivadh, Saudi Arabia

Background Congenital chloride diarrhea(CCD), A rare deadly autosomal recessive disorder of chronic diarrhea in infancy.

Methods Patients diagnosed with CCD between1986-2009 were studied. The demographic data, clinical findings and biochemical findings were collected and statistically analyzed.

Results Forty-three patients $(28 \mathrm{M} / 15 \mathrm{~F})$ had CCD. Fifteen patients $(35 \%)$ were diagnosed after one year of age (late referral or misdiagnosis as Bartter syndrome). Premature delivery in 24 cases (55.8\%). Polyhydramnios in 26 pregnancies. All patients were distributed among 19 families with 33 children being the outcome of consanguineous marriages. Intractable diarrhea was the presenting symptom in 40 patients (93\%), Biochemical data revealed: Serum potassium (1.3-4.1, mean $2.4 \mathrm{Mmol} / \mathrm{l})$, s. chloride (39-95, mean76.2Mmol/l), s.bicarbonate (22-54) meam-37.6 Mmol/). Fecal chloride (134 \pm 21.6 , mean \pm SD) (range 90-205). The fecal chloride over fecal sodium plus potassium ratio was $0.6(1.1 \pm 0.3$, mean $\pm \mathrm{SD})$ $(\mathrm{N} .=0.2)$. Associated disorders were: chronic renal failure $7(16 \%)$, congenital anomalies 8 (19\%), mental retardation4 (9.3\%) seizures 8 $(19 \%)$, and brain atrophy 4 (9\%). Complications were seen mostly among patients with late referral or poor compliance. At diagnosis, $35(81.4 \%)$ cases were below -2SD for weight for-age, 31 (72\%) for weight-for-height, and 31 (72\%) for height-for-age. Children under five years of age showed improvement in weight for height as compared with older children.

Conclusions CCD is a treatable cause of intractable diarrhea in infancy.

Awareness, early diagnosis and proper management are essential in preventing irreversible and long-term organ damage and a better outcome compared to those with late referrals.

CCD is to be considered in infants with severe persistent diarrhea where a high rate of consanguineous marriage prevails.

\section{LIVING DONOR LIVER TRANSPLANTATION FOR ALAGILLE SYNDROME: RECIPIENT CHARACTERISTICS AND OUTCOME IN A SINGLE CENTER}

doi:10.1136/archdischild-2012-302724.0163

MM Tiao', IF Huang'ㄹ. Liver Transplantation Team. 'Pediatrics, Kaohsiung Chang Gung Memorial Hospital and Chang Gung University College of Medicine; ${ }^{2}$ Pediatrics, Kaohsiung Veterans General Hospital, Kaohsiung, Taiwan R.O.C.

Background and aims Alagille syndrome (AGS) is rare in Taiwan. The aim of this study was to review our institutional experience with liver transplantation (LT) for AGS.

Methods We performed a retrospective analysis of transplant records of patients diagnosed as AGS and underwent LT between 1987 and 2010. Nine patients underwent living donor LT.

Results Cholestasis and characteristic faces were seen in all patients. Posterior embryotoxon was seen in 4/9 (44.4\%), butterfly vertebrae in $3 / 9(33.3 \%)$, heart defect (pulmonary stenosis in 2 ) in $3 / 9(33.3 \%)$, and renal disease in $2 / 9(22.2 \%)$ patients. Iminodiacetic acid scans showed no excretion of isotope into the bowel after 24 hours in $4 / 9(44.4 \%)$. A small gallbladder on ultrasonography was noted in $3 / 9(33.3 \%)$ and suggested a false diagnosis of biliary atresia. All underwent diagnostic laparotomy and liver biopsy. Liver biopsy showed characteristic features of paucity of interlobular bile ducts in all patients. Kasai portoenterostomy was not performed in any patient before being referred for LT. The mean age at time of LT was 4.6 years. The 5 -year overall survival rate after living donor LT was $88.9 \%$.

Conclusions Our conclusion is that the clinical features of AGS are informative. Histological confirmation is important in the diagnosis. These findings support the concept that infants with liver diseases warrant early referral to a specialist center.

\section{LINEAR GROWTH AFTER PEDIATRIC LIVER TRANSPLANTATION}

doi:10.1136/archdischild-2012-302724.0164

W Saleem, H Abdelrahman, A Elawwa, A Soliman. Pediatrics, Hamad Medical Center, Doha, Qatar

To determine growth patterns in a children undergoing liver transplantation, the outcomes of orthotopic liver transplantations performed in 10 children at Hamad General Hospital between October 2005 and October 2009 were reviewed. The mean age at transplantation was $27+/-30$ months; $80 \%$ of the children were females. The 